\title{
AN IDEAL CRITERION FOR TORSION FREENESS
}

\author{
MARK BRIDGER
}

\begin{abstract}
Auslander and Bridger have shown that, under conditions somewhat weaker than finite projective dimension, the "torsion freeness" properties of a module $M$ (e.g. being reflexive, being the $k$ th syzygy of another module) are determined by certain arithmetic conditions on the $\operatorname{Ext}^{i}(M, R)$. In this paper it is shown that a single ideal, the intersection of the annihilators of these modules, gives this same information. This ideal is then related to the Fitting invariants and invariant factors of $M$, and a computation is made of certain syzygies of a quotient of $M$ (by a regular $M$-sequence).
\end{abstract}

Introduction. In [2] it is shown that under certain reasonable hypotheses (somewhat weaker than having finite projective dimension), the torsion freeness properties of a module $M$ are determined by the grades of the modules $\operatorname{Ext}^{i}(M, R)$ for $i>0$. These numbers, in turn, depend only on the ideals $\mathfrak{A}_{i}=\operatorname{rad}\left[\operatorname{Ann} \operatorname{Ext}^{i}(M, R)\right]$. Denoting by $\gamma(M)$ the intersection of these ideals, the author has shown that $\gamma(M)$ likewise determines the torsion freeness properties of $M$. In this paper this result will be partially generalized, and an explicit calculation made exhibiting the torsion freeness properties $(\S 3)$. In $\S 2$ we relate $\gamma(M)$ to the Fitting invariants and invariant factors of $M$.

Unless otherwise specified, all rings will be commutative and noetherian and modules finitely generated (hence finitely presented).

1. The ideals $\beta(M)$ and $\gamma(M)$. Although $M$ is projective if and only if $\operatorname{Ext}^{1}(M,-)=0$, one need not test $\operatorname{Ext}^{1}(M,-)$ on all modules. Let us write $\Omega M=\Omega^{1} M=\operatorname{ker}(P \rightarrow M)$ where $P \rightarrow M$ is any map of a projective module $P$ onto $M$. If we agree to call two objects $A$ and $B$ projectively equivalent when $A+P \approx B+Q$ for projective objects $P$ and $Q$, then it is well known that the projective "equivalence class" of $\Omega M$ depends only on that of $M$ (see Lemma 10 below).

LEMMA 1. $M$ is projective if and only if $\operatorname{Ext}^{1}(M, \Omega M)=0$.

Received by the editors February 5, 1971.

AMS 1970 subject classifications. Primary 13C10, 13C15, $13 \mathrm{D} 05$.

Key words and phrases. Syzygies, projective equivalence, finite projective dimension, fitting invariants, invariant factors, extension functors, regular sequences, $k$-torsion freeness, grade.

c) American Mathematica: Society 1972 
Proof. From left to right the implication is clear; on the other hand, $\operatorname{Ext}^{1}(M, \Omega M)=0$ implies that the exact sequence $0 \rightarrow \Omega M \rightarrow P \rightarrow M \rightarrow 0$ splits, so $M$ (and $\Omega M$ ) are projective.

For future use, we define the $i$ th syzygy of $M, \Omega^{i} M$, recursively by: $\Omega^{i} M=\Omega\left(\Omega^{i-1} M\right) ; \Omega^{\circ} M=M$. While any two $i$ th syzygies of $M$ are projectively equivalent. it is unknown to this author whether a module projectively equivalent to an ith syzygy of $M$ is, in general, actually realizable as such.

Proposition 2. Let $x \in R, x \neq 0$. The following statements are equivalent:

(i) $M_{\{x\}}$ is $R_{\{x\}}$-projective (here $M_{\{x\}}$ is the module of fractions with respect to powers of $x$ ).

(ii) $M \rightarrow M$ given by multiplication by $x^{i}$ factors through a projective for $i$ sufficiently large.

(iii) $x^{i} \operatorname{Ext}^{n}(M,-)=0$ for $n>0$ and $i$ sufficiently large.

(iv) $x^{i} \operatorname{Ext}^{1}(M, \Omega M)=0$ for $i$ sufficiently large.

Proof. Suppose (i) holds; let $F \stackrel{\eta}{\rightarrow} M \rightarrow 0$ be exact with $F$ projective. The epimorphism $[\eta / 1]: F_{\{x\}} \rightarrow M_{\{x\}}$ has a splitting which, since $M$ is finitely presented, is of the form $\left[x \mid x^{t}\right]$ where $\alpha: M \rightarrow F$. Thus,

$$
[\eta / 1]\left[\alpha / x^{t}\right]=\text { identity of } M_{\{x ;} \text {. }
$$

We conclude that $x^{j}(\eta \circ x)=x^{j} x^{t}$ for some $j \geqq 0$. Thus, for $i=j+t$ we have the following commutative diagram:

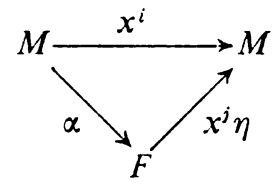

which verifies (ii).

Now suppose that (ii) holds; then the diagram

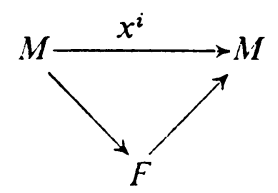

induces

giving (iii).

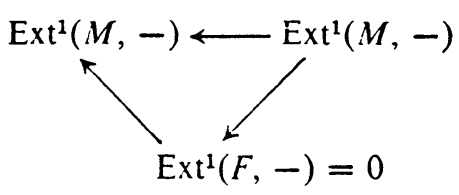

The implication (iii) $\Rightarrow$ (iv) is clear. 
Finally, if (iv) holds then

$$
0=\left(\operatorname{Ext}_{R}^{1}(M, \Omega M)\right)_{\{x\}}=\operatorname{Ext}_{R_{\{x\}}}^{1}\left(M_{\{x\}}, \Omega_{\{x\}} M_{\{x ;}\right)
$$

(forming syzygies is easily seen to commute with taking modules of fractions). The lemma now applies, so (i) holds.

Following the lead of this proposition, we have:

Definition 3. $\beta(m)=\left\{x \in R \mid M_{\{x\}}\right.$ is $R_{\{x ;}$-projective $\}$ (note that $\beta(M)$ as here defined is the radical of the ideal $\beta(M)$ defined in [1]).

Now if $M \neq 0$ happens to have finite projective dimension, then pd $M=$ $\sup \left\{i \mid \operatorname{Ext}^{i}(M, R) \neq 0\right\}$; in particular, $M$ is projective if and only if $\operatorname{Ext}^{i}(M, R)=0$ for all $i>0$. If we put $\mathfrak{A}_{i}=\operatorname{rad}\left(\operatorname{Ann} \operatorname{Ext}^{i}(M, R)\right)$ and $\gamma(M)=\bigcap_{i>0} \mathfrak{A}_{i}$, then we have:

Proposition 4. If pd $M<\infty$ then $\beta(M)=\gamma(M)$.

(This is an easy consequence of the above remarks and the fact that, for modules having finitely generated projective resolutions, taking Ext commutes with taking modules of fractions.)

\section{Relation to invariant factors and Fitting invariants.}

Definition 5. Given a presentation $R^{m} \stackrel{\Delta}{\rightarrow} R^{n} \rightarrow M \rightarrow 0$, the $p$ th Fitting invariant $F_{p}(M)$ is the ideal generated by the $(n-p+1) \times(n-p+1)$ minors of the matrix of $\Delta$. (This is shown to depend only on $M$.)

Definition 6. $\quad \alpha_{p}(M)=\mathrm{Ann}\left(\bigwedge^{p} M\right)$ is called the $p$ th invariant factor of $M$.

The following fact may be found in [3].

Proposition 7. For each $p, F_{p}(M)$ and $\alpha_{p}(M)$ have the same radicals.

Lemma 8. Let $\bar{\alpha}(M)=\bigcap_{\alpha_{i}(M) \neq 0} \alpha_{i}(M)$; then $\bar{\alpha}(M) \subset \beta(M)$.

Proof. Let $x$ be in $\bar{\alpha}(M)$. It suffices to prove that $M_{\wp}$ is $R_{\wp}$ projective for each prime $\wp$ not containing $x$ (since these correspond to the primes of $\left.R_{\{x\}}\right)$. Suppose then that $x \notin \wp$ and observe that over the ring $R_{\wp}$ we have: $\operatorname{Ann}\left(\bigwedge^{p} M_{\wp}\right)=\alpha_{p}(M) \otimes R_{\wp}$. This last ideal is zero if $\alpha_{p}(M)=0$ and all of $R_{\wp \rho}$ if $\alpha_{p}(M) \neq 0$, since $x$ is then in $\alpha_{p}(M)$. Thus, the invariant factors of $M_{\wp}$ over $R_{\wp}$ are either zero or the whole ring. However, in [1, Proposition 2.3] it is shown that if $n=$ exterior rank $E=\sup \left\{i \mid \bigwedge^{i} E \neq 0\right\}$ then (over local rings) $E$ is free if and only if $\alpha_{n}(E)=0$. Applying this to $M_{\wp}$ gives our results.

Proposition 9. If $R$ is a domair: or pd $M<\infty$ then $\operatorname{rad} \bar{\alpha}(M)=\beta(M)$.

Proof. One inclusion is the above lemma. To prove $\beta(M) \subset \operatorname{rad} \bar{\alpha}(M)$ we use a slight modification of the proof given in [1] for the case $R$ a domain. Suppose $\alpha_{p}(M) \neq 0$ and $x: M \rightarrow M$ factors through a projective $F$. 
We may assume $F$ is free since it is easily shown that a map $A \rightarrow B$ factors through a projective if and only if it factors through any projective mapping onto $B$. We obtain first a commutative diagram:

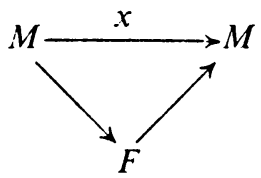

which yields another:

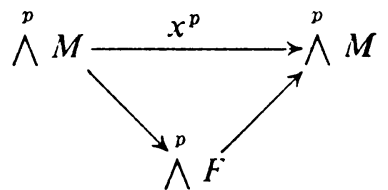

Since $F$ is free, it suffices to prove that grade $\bigwedge^{p} M>0$, this being equivalent to $\operatorname{Hom}\left(\bigwedge^{p} M\right.$, Free $)=0$. When $R$ is a domain, the grade condition holds since $\alpha_{p}(M) \neq 0$ means that $\alpha_{p}(M)=$ Ann $\wedge^{p} M$ contains an $R$-regular element. Let us go then to the case $\mathrm{pd} M<\infty$. The question of grade being a local one, we may suppose $R$ to be local. Therefore $M$ has a finite free resolution. For such a module it is known [5] that the Fitting invariants are either zero (for $p \leqq \chi(M)=$ Euler characteristic of $M$ ) or contain a nonzero divisor $(p>\chi(M))$. Since $\alpha_{p}(M)$ and $F_{p}(M)$ have the same radicals, as was pointed out in Proposition 7 above, we see that $\alpha_{p}(M)$ contains an $R$-regular element; therefore grade $\bigwedge^{p} M>0$ and so multiplication by $x^{p}$ is zero on $\wedge^{p} M$.

3. A calculation of syzygies. As was pointed out above, the syzygies of a module are determined only up to projective equivalence. One way of expressing this fact is by the following well-known criterion:

LemMA 10. $A$ is projectively equivalent to $B$ if and only if $\operatorname{Ext}^{1}(A,-)$ and $\operatorname{Ext}^{1}(B,-)$ are naturally isomorphic functors. In particular, $A$ is projectively equivalent to $\Omega^{n} M$ if and only if $\operatorname{Ext}^{1}(A,-) \approx \operatorname{Ext}^{n+1}(M,-)$.

We now come to the main result of this section.

THEOREM 11. Let $\left(x_{1}, \cdots, x_{k}\right)$ be an M-regular sequence contained in $\beta(M)$. Then there is a positive integer $n$ such that, if $y_{i}=x_{i}^{n}$, then

$$
\Omega^{k}\left(M /\left(y_{1}, \cdots, y_{k}\right) M\right) \sim \sum_{i=0}^{k}\left(\begin{array}{l}
k \\
i
\end{array}\right) \cdot \Omega^{i} M .
$$

(Here $\sim$ denotes projective equiralence and $t \cdot N$ the direct sum of $t$ copies of $N$.) 
Proof. By Proposition 2 there is, for each $i$, an integer $n(i)$ such that $x_{i}^{n(i)}: M \rightarrow M$ factors through a projective $P$. Let $n=\max n(i)$.

Note that multiplication by $x_{i}^{n}$ on $\Omega^{s} M$ also factors through a projective $\left(v i z . \Omega^{s} P\right)$ for each $s>0$.

We proceed by induction. When $k=0$ there is nothing to prove. Suppose then the theorem hoids for an integer $k \geqq 0$, and let $x_{1}, \cdots, x_{k+1}$ be an $M$-regular sequence contained in $\beta(M)$. Then we have

$$
\Omega^{k}\left(M /\left(y_{1}, \cdots, y_{k}\right) M\right) \sim \sum_{i=0}^{k}\left(\begin{array}{l}
k \\
i
\end{array}\right) \cdot \Omega^{i} M=(\operatorname{Id}+\Omega)^{k}(M) .
$$

To simplify notation let $\bar{M}=M /\left(x_{1}^{n}, \cdots, x_{k}^{n}\right) M, x=x_{k+1}$. Consider the exact sequence

$$
0 \longrightarrow \bar{M} \stackrel{x^{n}}{\longrightarrow} \bar{M} \longrightarrow \bar{M} / x^{n} \bar{M} \longrightarrow 0 .
$$

Note that $\bar{M} / x^{n} \bar{M}=M /\left(x_{1}^{n}, \cdots, x_{k}^{n}, x_{k+1}^{n}\right) M$. Part of the long exact sequence for Ext gives:

$$
\begin{aligned}
\operatorname{Ext}^{k+1}(\bar{M},-) & \stackrel{x^{n}}{\longrightarrow} \operatorname{Ext}^{k+1}(\bar{M},-) \longrightarrow \operatorname{Ext}^{k+2}\left(\bar{M} / x^{n} \bar{M},-\right) \\
& \longrightarrow \operatorname{Ext}^{k+2}(\bar{M},-) \stackrel{x^{n}}{\longrightarrow} \operatorname{Ext}^{k+2}(\bar{M},-)
\end{aligned}
$$

Now multiplication by $x^{n}$ on $\Omega^{k} \bar{M}$ factors through a projective by the induction hypothesis. Since $\operatorname{Ext}^{k+i}(\bar{M},-) \approx \operatorname{Ext}^{i}\left(\Omega^{k} \bar{M},-\right)$ we see that $x^{n} \operatorname{Ext}^{k+i}(\vec{M},-)=0$ and we obtain the short exact sequence:

$$
0 \rightarrow \operatorname{Ext}^{1}\left(\Omega^{k} \bar{M},-\right) \rightarrow \operatorname{Ext}^{1}\left(\Omega^{k+1}\left(\bar{M} / x^{n} \bar{M}\right),-\right) \rightarrow \operatorname{Ext}^{1}\left(\Omega^{k+1} \bar{M},-\right) \rightarrow 0 .
$$

A short exact sequence of Ext functors is, however, known to split (see [4]). Therefore

$$
\operatorname{Ext}^{1}\left(\Omega^{k+1}\left(\bar{M} / x^{n} \bar{M}\right),-\right) \approx \operatorname{Ext}^{1}\left(\Omega^{k} \bar{M}+\Omega^{k+1} \bar{M},-\right) .
$$

By Lemma 10 above $\Omega^{k+1}\left(\bar{M} / x^{n} \bar{M}\right) \sim \Omega^{k} \bar{M}+\Omega^{k+1} \bar{M}=(\operatorname{Id}+\Omega)\left(\Omega^{k} \bar{M}\right)=$ $(\operatorname{Id}+\Omega)(\operatorname{Id}+\Omega)^{k}(M)=\sum_{i=0}^{k+1}\left(\begin{array}{c}k+1 \\ i\end{array}\right) \Omega \Omega^{i} M$.

This completes the induction and the proof.

4. Torsion freeness. In this section we find a (partial) converse to Theorem 1. Let us recall first the notion of $k$-torsion freeness; for further details see [2]. For a module $M$, choose a presentation $P_{1} \rightarrow P_{0} \rightarrow M \rightarrow 0$ and write $D M=\operatorname{coker}\left(P_{0}^{*} \rightarrow P_{1}^{*}\right)$ where $P^{*}=\operatorname{Hom}(P, R)$. Then $D M$ depends (up to projective equivalence) only on $M$.

Definition 12. $M$ is $k$-torsion free if $\operatorname{Ext}^{i}(D M, R)=0$ for $1 \leqq i \leqq k$.

The reason for this definition is the following exact sequence:

$$
0 \rightarrow \operatorname{Ext}^{1}(D M, R) \rightarrow M \rightarrow M^{* *} \rightarrow \operatorname{Ext}^{2}(D M, R) \rightarrow 0
$$


so 1-torsion freeness is equivalent to torsion freeness, while 2-torsion freeness is equivalent to reflexivity.

REMARK. If $N$ is a direct summand of $M$ and $M$ is $k$-torsion free, then so is $N$. In particular, if $N$ is projectively equivalent to a $k$-t.f. module, $N$ is itself $k$-t.f.

The proof of the following may be found in [2]:

Proposition 13. Consider the properties:

(a) $M$ is $k$-torsion free.

(a') There is an exact sequence $0 \rightarrow M \rightarrow P_{k-1} \rightarrow \cdots \rightarrow P_{0}$ where the $P_{i}$ are projective and $P_{0}^{*} \rightarrow \cdots \rightarrow P_{k-1}^{*} \rightarrow M^{*} \rightarrow 0$ is exact.

(b) There is an exact sequence $0 \rightarrow M \rightarrow P_{k-1} \rightarrow \cdots \rightarrow P_{0}$ where the $P_{i}$ are projective.

(c) $M$ is projectively equivalent to $\Omega^{k} M$ for some $N$.

(d) Every $R$-regular sequence of length $\leqq k$ is $M$-regular.

Then the following implications hold: $(\mathrm{a}) \Leftrightarrow\left(\mathrm{a}^{\prime}\right) \Rightarrow(\mathrm{b}) \Rightarrow(\mathrm{c}) \Rightarrow$ (d). If $\mathrm{pd} M<$ $\infty$ then each of these is equivalent to

(e) grade $\operatorname{Ext}^{i}(M, R) \geqq i+k$ for each $i>0$.

Remark. The hypothesis pd $M>\infty$ may actually be replaced by the weaker condition $G\left(R_{\wp}\right)-\operatorname{dim} M_{\S 0}<\infty$ for each prime $\wp$; details may be found in [2]. We now use the equivalence of these properties ( $\operatorname{pd} M<\infty$ ) to prove

THEOREM 14. Suppose pd $M<\infty$. Then $M$ is a $k$-syzygy (equivalently: $M$ is $k$-torsion free) if and only if $\gamma(M)$ contains an $M$-regular sequence of length $k$.

Proof. Suppose $\gamma(M)$ contains an $M$-regular sequence of length $k$. Since $\gamma(M)=\beta(M)$ in this case-Proposition 4-we see that $M=\Omega^{\circ} M$ is a direct summand of $\Omega^{k}\left(M /\left(y_{1}, \cdots, y_{k}\right) M\right)$ by Theorem 11. By Proposition 13 this last module is $k$-torsion free. Finally, by the first remark of this section, $M$ is $k$-t.f., being a direct summand of a $k$-t.f. module.

Conversely, suppose $M$ is $k$-t.f. Then grade $\operatorname{Ext}^{i}(M, R) \geqq i+k$ for each $i>0$. Let $\wp$ be any prime containing $\gamma(M)=\bigcap_{i>0} \operatorname{rad}\left[\operatorname{Ann} \operatorname{Ext}^{i}(M, R)\right]$. Then $\wp$ contains Ann $\operatorname{Ext}^{i}(M, R)$ for some $i$. We conclude that $\rho$ contains an $R$-regular sequence of length (at least) $k$ by the grade condition on $\operatorname{Ext}^{i}(M, R)$. Since $M$ is $k$-torsion free, Proposition 13 shows that such a sequence is $M$-regular as well; $\wp$ being arbitrary, $\wp \supset \gamma(M)$, we conclude that $\gamma(M)$ contains an $M$-regular sequence of length $k$. This completes the proof.

\section{REFERENCES}

1. M. Auslander and D. A. Buchsbaum, intariant factors and two criteria for projectivity of modules, Trans. Amer. Math. Soc. 104 (1962), 516-522. MR 28 \#1215. 
2. M. Auslander and M. Bridger, Stable module theory, Mem. Amer. Math. Soc. No. 94 (1969). MR 42 \#4580.

3. D. Buchsbaum, Complexes associated with the minors of a matrix, Brandeis University, Waltham, Mass. (mimeographed notes).

4. P. J. Hilton and D. Rees, Natural maps of extension functors and a theorem of R. G. Swan, Proc. Cambridge Philos. Soc. 57 (1961), 489-502. MR 23 \#A1689.

5. I. Kaplansky, Commutative algebra, Allyn and Bacon, Boston, Mass., 1970. MR 40 \#7234.

Department of Mathematics, Northeastern University, Boston, MassachuSETTS 02115 